\section{Time to Change: Supporting Sexual and Gender Minority People-An Underserved, Understudied Cancer Risk Population}

\author{
Juno Obedin-Maliver, MD, MPH, MAS
}

Sexual and gender minorities (SGMs), which include but are not limited to lesbian, gay, bisexual, transgender, and queer (LGBTQ) people, likely represent at least 4\% of the current US population, ${ }^{1}$ and will encompass 2 to 6 million people over age 65 years by 2030. ${ }^{2}$ As do people of every demographic, they get cancer, and risk increases with age. The absence of sexual orientation and gender identity (SOGI) metrics in the US Census and national demographic and health studies and cancer registries like SEER, leaves us largely ignorant of the prevalence and incidence of cancer among SGM people. What is now indisputable is that SGM people face significant health and healthcare disparities that translate to important differences in cancer risk factors and screening, ${ }^{3-7}$ morbidity, ${ }^{8,9}$ and mortality. ${ }^{10}$ What data exist suggest a differential prevalence of cancer overall among bisexual and lesbian women compared with straight women, likely higher prevalence of breast cancer in those same groups, and anal cancer among men who have sex with men and gay men. ${ }^{11}$ For significant subpopulations under the SGM umbrella (eg, transgender individuals) no reliable data on cancer incidence and prevalence patterns exist. Although the NIH recently declared SGM people a health disparity population for research, interest and investment in SGM health has been unbalanced. Only 1.8\% of SGM-focused research addresses cancer; $75 \%$ is focused on HIV/AIDs. ${ }^{11}$

The pervasive legal, social, and medical discrimination faced by SGM populations creates persistent psychological stress states with multiple health implications, termed "minority stress." ${ }^{2}$ Experienced as stigma, discrimination, and even abject violence, minority stress affects every aspect of individuals' lives including presence or severity of cancer risk factors (eg, smoking, obesity, infections like HIV and HPV), likelihood of timely screening, equitable diagnosis, and administration of effective treatment. ${ }^{13}$ Our burden as clinicians and investigators is to care for this population throughout the cancer care continuum, despite continued gaps in research and health population statistics.

For example, consider cervical cancer. Cervical cancer is universally described as a cancer of women alone. What is obscured is the distinction between sex and gender. Cervical tissue is present in individuals who were born with a cervix. Sex is the set of genetic, anatomic, and physiologic characteristics of males and females of our species, whereas gender represents the social, cultural, and behavior norms associated with being a man, woman, or another gender. There are many men who were born with a uterus and cervix..$^{14,15}$ Furthermore, cervical cancer screening frequency and outcomes are different between cisgender (ie, non-transgender) women and transgender men (those who identify as men but were assigned female sex at birth). ${ }^{7}$ Differences in cervical cancer screening outcomes by gender seem to be related to exposure to exogenous testosterone and to the system-wide, psychosocial, and physical experiences that hinder obtaining medical care and timely screening. ${ }^{16}$

Imagine the experience of a transgender man seeing a CDC bulletin board saying, "The Pap test is recommended for all women between 21 and 65." Insulted by the misgendering but proactive about his health, he undergoes cervical cancer screening. Unfortunately, he is told he has cervical cancer and must present for treatment at the "Women's Cancer Center." While waiting to see the gynecologic oncologist,

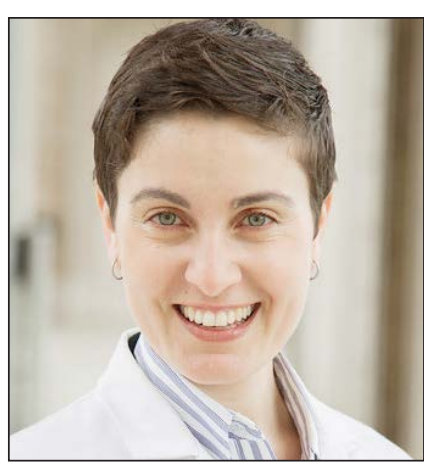

Juno Obedin-Maliver, MD, $\mathrm{MPH}, \mathrm{MAS}$

Juno Obedin-Maliver, MD, MPH, MAS, is an Assistant Professor in the Department of Obstetrics, Gynecology \& Reproductive Sciences at the University of California, San Francisco. She also serves as the Chief of Gynecology at the San Francisco Veterans Affairs Medical Center. Academically she focuses on promoting the health and well-being of sexual and gender minorities, which include but are not limited to lesbian, gay, bisexual, transgender, and queer (LGBTQ) people. Dr. ObedinMaliver is the Co-Director of The PRIDE Study (pridestudy.org), an online prospective longitudinal cohort of sexual and gender minority individuals based at University of California, San Francisco. 
he ponders the clinic banner that says "Excellence in the Care of Women." The awkwardness continues as Mr. Doe sees a gynecologic oncologist who admits she's never cared for a transgender man before, uses incorrect pronouns, calls Mr. Doe by his "dead" name, and stumbles to understand why he describes himself as straight when introducing his cisgender female fiancé because the oncologist does not understand the difference between sexual orientation and gender identity. Eventually, still reeling from a cancer diagnosis, Mr. Doe has to book a hysterectomy in the Women's Surgical Center but has not had a chance to mourn his loss of an ability to carry children (this was never discussed in treatment outcomes), his fear of surgery, or facing his own mortality.

In this issue of JNCCN, Hudson et al's report (page 1379) brings to the fore the "ignored epidemic" of cancer among SGM people. I applaud the report for its success in raising awareness of potential gaps in current NCCN Clinical Practice Guidelines in Oncology (NCCN Guidelines); however, the authors' brief questionnaire to NCCN Panel Members is unable to plumb the members' understanding of and future intentions for "addressing LGBTQ medical or psychological issues." Further, the grammatically and sociologically challenging questions Hudson et al raise to NCCN Panel Members as to whether "sexual orientation" or "gender identity" are relevant demographics for the focus of the panel deserves some unpacking. "Sexual orientation" and "gender identity" are not populations, but rather metrics. Everyone has a sexual orientation and a gender identity. What the authors may have meant to ask, and likely what the respondents answered, was, "Are sexual minority populations, or gender minority populations, a relevant demographic for the focus of your panel?" Answering that question is hard given the lack of current data, but frankly there is no cancer type in which SGM people are not a relevant population, because SGM people will get every type of cancer and need sensitive patient-centered cancer care.

Studies such as this one show us that understanding the relationship between being SGM and the cancer continuum is a nascent field. The call for data and understanding the experience of SGMs in every cancer research study and registry is timely and mirrors that of a recent ASCO Position Statement. ${ }^{17}$ Frankly, without these data, we are flying blind in differential impact of cancer diagnosis, treatment, and outcomes on this community.

Although the oncology community has a long way to go to understand the full spectrum of cancer care needs for the SGM community, concrete steps can be taken to understand and address the needs of this diverse community. First, we can't fix what we don't understand. Therefore, systematic inclusion of sexual orientation and gender identity measures in all clinical and investigation contexts is key. To this end, all clinical sites should comply with the voluntary, but important, mandate for collection of sexual orientation and gender identity of meaningful use. ${ }^{18}$ All cancer care providers and comprehensive cancer centers should modify intake and data collection forms to assess sexual orientation, gender identity, and sex assigned at birth so we can start to understand risk, incidence, prevalence, treatment, and prognosis indicators.

Second, we must create and maintain clinical environments that care for the whole person. Beyond assessing sexual orientation, gender identity, and sex assigned at birth, we need to understand what to do with that information both socially and clinically. This means education and training for all personnel (including patient greeter, front desk staff, billing department, and all clinical providers) and ensuring that the physical environment and services provided reflects true diversity. This means assessing signage, magazines and posters, bathroom access, nondiscrimination policies, and symptom support centers and patient advocacy groups — do they reflect and serve all people? Good tools to assess and modify clinical environments and training can be found through The Fenway Institute (https://www.lgbthealtheducation.org), and 
comprehensive resources for cultural competency training for providers and support for SGM people with cancer can be found through the National LGBT Cancer Network (https://cancer-network.org).

Third, to move the field forward, we need to understand how SGM peoples' experience at every step of the cancer care continuum might be the same or different than that of heterosexual and cisgender peers. However, this can only be done via research and clinical trials, which often exclude SGM people. ${ }^{19}$ National SGM research cohorts like The PRIDE Study (pridestudy.org) are one important step, but true health equity will only come when every cancer research study includes these demographics.

Finally, the NCCN Guidelines should make modifications and herald an awareness that promotes care for all cancer survivors. For example, NCCN panels should enhance discussion of differences in risk factors, screening, and treatment among populations to include SGM people. This follows prior models like that of the NCCN Guidelines for Prostate Cancer regarding the discussions of the experiences of African American men versus Caucasian American men. For example, that same guideline could discuss prostate cancer risk and screening for transgender women (individuals who were assigned male sex at birth but identify as women) who have undergone vaginoplasty, noting that prostate examinations are best performed via the neovagina, and discussing how estrogen administration alters prostate-specific antigen (PSA) levels. On a broader scale, altering NCCN Guidelines language for relevance to people of all genders and sexual orientations by using sex-based anatomy and not assuming heterosexual relationships or sexual practices will enhance patient comfort and promote understanding of this underserved community.

However, since change to the NCCN Guidelines may take time, commit to immediate local action. Individual clinicians can affect change immediately in patient encounters, in clinic systems, research metrics, and in advocacy at the national level. Simple steps taken by every oncologist, in whatever domain you practice, can make a world of difference in meeting the needs of SGM people-an often-neglected cancer risk community.

\section{References}

1. Gates GJ. How Many People Are Lesbian, Gay, Bisexual and Transgender? 2011: The Williams Institute. Available at https://williamsinstitute.law.ucla.edu/wp-content/uploads/Gates-How-Many-People-LGBT-Apr-2011.pdf. Accessed October 19, 2017.

2. Fredriksen-Goldsen KI, Muraco A. Aging and sexual orientation: a 25-year review of the literature. Res Aging 2010;32:372-413.

3. Collins TW, Grineski SE, Morales DX. Environmental injustice and sexual minority health disparities: a national study of inequitable health risks from air pollution among same-sex partners. Soc Sci Med 2017;191:38-47.

4. Lunn MR, Cui W, Zack MM, et al. Sociodemographic characteristics and health outcomes among lesbian, gay, and bisexual U.S. adults using Healthy People 2020 leading health indicators. LGBT Health 2017;4:283-294.

5. Agenor M, Muzny CA, Schick V, et al. Sexual orientation disparities in the utilization of sexual health services in a national probability sample of U.S. women. Prev Med 2017;95:74-81.

6. Peitzmeier SM, Khullar K, Reisner SL, Potter J. Pap test use is lower among female-to-male patients than nontransgender women. Am J Prev Med 2014;47:808-812.

7. Peitzmeier SM, Reisner SL, Harigopal P, Potter J. Female-to-male patients have high prevalence of unsatisfactory Paps compared to non-transgender females: implications for cervical cancer screening. J Gen Intern Med 2014;29: 778-784.

8. Boehmer U, Glickman M, Winter M, Clark MA. Long-term breast cancer survivors' symptoms and morbidity: differences by sexual orientation? J Cancer Surviv 2013;7:203-210.

9. Boehmer U, Miao X, Linkletter C, Clark MA. Health conditions in younger, middle, and older ages: are there differences by sexual orientation? LGBT Health 2014;1:168-176.

10. Lehavot K, Rillamas-Sun E, Weitlauf J, et al. Mortality in postmenopausal women by sexual orientation and veteran status. Gerontologist 2016;56(Suppl 1):S150-162.

11. National Institutes of Health Sexual and Gender Minority Research Coordinating Committee. NIH FY 2016-2020 Strategic Plan to Advance Research on the Health and Well-being of Sexual and Gender Minorities. Bethesda, MD: National Institutes of Health; 2015. Available at https://www.edi.nih.gov/sites/default/files/EDI_Public_files/ sgm-strategic-plan.pdf. Accessed October 20, 2017. 
Obedin-Maliver

12. Hatzenbuehler ML, Bellatorre A, Lee $Y$, et al. Structural stigma and all-cause mortality in sexual minority populations. Soc Sci Med 2014;103:33-41.

13. Makadon HJ, Mayer KH, Potter J, Goldhammer H. Fenway guide to lesbian, gay, bisexual, and transgender health, 2nd ed. Philadelphia, PA: American College of Physicians; 2015.

14. Conron KJ, Scott G, Stowell GS, Landers SJ. Transgender health in Massachusetts: results from a household probability sample of adults. Am J Public Health 2012;102:118-122.

15. Grant JM, Mottet LA, Tanis J, et al. Injustice at Every Turn: A Report of the National Transgender Discrimination Survey. Washington, DC: National Center for Transgender Equality and National Gay and Lesbian Task Force; 2011. Available at http://www.thetaskforce.org/injustice-every-turn-report-national-transgender-discriminationsurvey/. Accessed October 20, 2017.

16. Potter J, Peitzmeier SM, Bernstein I, et al. Cervical cancer screening for patients on the female-to-male spectrum: a narrative review and guide for clinicians. J Gen Intern Med 2015;30:1857-1864.

17. Griggs JJ, Maingi S, Blinder V, et al. American Society of Clinical Oncology position statement: strategies for reducing cancer health disparities among sexual and gender minority populations. J Clin Oncol 2017;35:22032208.

18. Cahill SR, Baker $K$, Deutsch MB, et al. Inclusion of sexual orientation and gender identity in stage 3 meaningful use guidelines: a huge step forward for LGBT health. LGBT Health 2016;3:100-102.

19. Egleston BL, Dunbrack RL Jr, Hall MJ. Clinical trials that explicitly exclude gay and lesbian patients. N Engl J Med 2010;362:1054-1055. 